Int. J. Dev. Biol. 51: 473-481 (2007)

doi: $10.1387 /$ ijdb.072334ts

\title{
Expression and functions of FGF ligands during early otic development
}

\author{
THOMAS SCHIMMANG* \\ Institute for Molecular Biology and Genetics, University of Valladolid and Spanish Research Council (CSIC), Valladolid, Spain
}

\begin{abstract}
Classical studies have postulated the action of an endomesodermal signal initiating inner ear induction, subsequently followed by a neural tube-derived signal to complete the process of otic placode formation in the surface ectoderm. Members of the Fibroblast growth factor (FGF) gene family have been implicated in these processes. In this review, expression analysis and recent experimental evidence for candidate inner ear FGF ligands during inner ear induction is discussed. Careful examination of the spatiotemporal expression patterns of different FGFs during inner ear induction reveals that the sequential appearance of FGF members in the endoderm and/or mesoderm is followed by expression in the posterior hindbrain in all vertebrate species analysed to date. Experimental manipulations have demonstrated the sufficiency and/or necessity of some FGFs during different steps of inner ear induction in vitro and in vivo. Combining the advantages of the molecular tools and approaches available in different experimental systems such as zebrafish, chicken or mouse will eventually lead to a complete understanding of how FGFs control inner ear induction in vertebrates.
\end{abstract}

KEY WORDS: inner ear, otic placode, fibroblast growth factor, otic induction

Inner ear induction is already initiated during gastrulation by endomesodermal tissue which comes to underlie competent ectoderm. During neurulation, a second inducing neural signal from the presumptive hindbrain reinforces and maintains inner ear induction. The initial classical embryology experiments, addressing the sufficiency and necessity of different tissues during inner ear induction, have in more recent times been combined with molecular probes and tools to begin to build a molecular framework explaining different steps of inner ear induction (Baker and Bronner-Fraser, 2001; Noramly and Grainger, 2002; Groves, 2005). Members of the Fibroblast growth factor (FGF) gene family are among the prime candidates to control inner ear induction since they show a spatiotemporal expression pattern consistent with playing a role during this process. Secondly, their inductive capacities and necessity during embryonic patterning and the formation of various organ systems underscores their potential to also participate during the early phases of inner ear formation (Reuss and von Bohlen und Halbach, 2003; Bottcher and Niehrs, 2005; Thisse and Thisse, 2005a). In this review the expression patterns of FGFs and the recent experimental evidence for their participation during inner ear induction is reviewed.

\section{Expression of FGFs during inner ear induction}

In this first section the spatiotemporal expression patterns of FGFs during inner ear induction in different vertebrate models is described. Following the experimental evidence that a first inductive signal for otic placode formation is present in the endomesoderm we refer to this phase as the initiation of induction (Fig. 1A,D). The second phase of the induction process is initiated by a neural signal from the developing hindbrain and is complete by the onset of placode formation (Fig. 1B,E). Finally, we refer to FGFs expressed at the moment when the otic placode has completed its formation and starts its invagination (Fig. 1C,G). During inner ear induction in the chicken these three phases roughly correspond to the periods before (Fig. 1A) and after (Fig. 1B) the specification of part of the preplacodal domain to form the otic placode and after the commitment to the otic fate (Fig. 1C; Groves and Bronner-Fraser, 2000; Bailey and Streit, 2006)

\section{Chicken FGF expression}

During initiation of inner ear induction in chicken embryos FGF8 and FGF19 are the first FGF family members detected at the 0 somite stage (ss, stage HH6 after Hamburger and Hamilton, 1992; Ladher et al., 2000; Ladher et al., 2005). Fgf8is expressed in the endoderm whereas Fgf19is detected in mesoderm that underlies the preplacodal ectoderm (Fig. 1A). The exact temporal order of expression of both FGFs is at present unclear although one study defines the onset of Fgf19 expression slightly later at the 1ss (HH7; Kil et al., 2005). At $\mathrm{HH} 7$ also Fgf3expression is first detected in the mesoderm where it is coexpressed with Fgf19(Fig. 1A; Kil etal., 2005). At this stage Fgf3

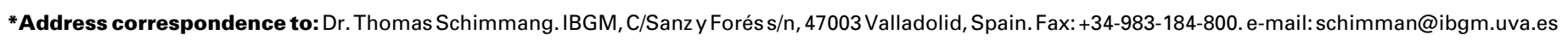

0214-6282/2007/\$30.00

(c) UBC Press

Printed in Spain

www.intjdevbiol.com 
transcripts have also been described in the unsegmented hindbrain just anterior to the first somite (Mahmood et al., 1995), although this domain may correspond to the Fgf3 hybridisation signal from the underlying mesoderm (Kil etal., 2005). Expression of Fgf8, Fgf3 and Fgf19 are maintained in the endoderm and mesoderm, respectively, during the following phase of induction until 7ss (HH9) at around the stage when the otic placode can first be visualized (Fig. 1B; Ladher et al., 2000; Karabagli et al., 2002; Brown et al., 2003; Kil et al., 2005). During this period, additional expression domains for Fgf3and Fgf19are now also apparent in the endoderm and developing hindbrain. In the pharyngeal endoderm Fgf8 is accompanied almost simultaneously by Fgf3 (5ss) and Fgf19(6ss) expression (Mahmood et al., 1995; Wright et al., 2004; Ladher et al., 2005). Even earlier, Fgf3 is clearly detected in the hindbrain from the $3-4$ ss onwards and is thus turned on before the earliest described otic placode marker, Pax2, is specified at the 4-5ss (Groves and Bronner-Fraser, 2000; Kil et al., 2005). Upon morphogenesis of hindbrain rhombomeres ( $r$ ) at 7ss, Fgf3expression is observed in r4 and r5 (Mahmood et al., 1995). Finally, from the 5ss until 9ss, Fgf19is transiently observed in the posterior ventral hindbrain (Ladher et al., 2000; Wright et al., 2004; Kil et al., 2005). Interestingly, expression of FGF family members has not been detected in the preplacodal ectoderm so far (Fig. 1B).

When the otic placode has formed and starts to invaginate, the Fgf3 expression domain in the hindbrain is maintained but also extends to include r6 at 10ss (Fig. 1C, Mahmood et al., 1995). Likewise, at this stage Fgf3, Fgf8 and Fgf19 expression is still observed in the pharyngeal endoderm (Hidalgo-Sanchez et al., 2000; Ladher et al., 2000; Adamska et al., 2001; Karabagli et al., 2002; Stolte et al., 2002; Wright et al., 2004). Moreover, the otic placode itself now shows abundant transcripts for Fgf10, as well as more weak expression for Fgf8(Adamska etal., 2001; Karabagli et al., 2002).

\section{Mouse FGF expression}

In mouse embryos Fgf8 is already detected at embryonic day 7 (E7) in the heart mesoderm underlying the neural plate (Ladher et al., 2005), although the relevance of this Fgf8 expression domain for otic induction is uncertain (Fig. 1D). At E8 (0ss) Fgf8 expression is detected in the splachnic mesoderm and at the 3ss all mesenchyme underneath the preplacodal ectoderm shows Fgf8 transcripts (Ladher et al., 2005). The potential influence of this expression domain on otic induction is underlined by the finding that Fgf10 expression is also observed in this mesenchyme from the 0ss stage onwards (Fig. 1D; Alvarez et al., 2003; Wright and Mansour, 2003a). During E8, Fgf10 expression is maintained in the mesenchyme whereas Fgf8transcripts are now also transiently observed in the preplacodal ectoderm and the pharyngeal endoderm from the 4 to 8ss (Fig. 1E; Crossley and Martin, 1995; Ladher et al., 2005). Around the same time (3ss), Fgf3 expression is first detected in the hindbrain and preplacodal ectoderm (Wright and Mansour, 2003a). Fgf3 expression is initially detected as a stripe in the presumptive posterior hindbrain, but from the 5 ss onwards broadens rostrally and reaches the level of $\mathrm{r} 1$ (Mahmood et al., 1996; McKay et al., 1996; Alvarez et al., 2003; Wright and Mansour, 2003a; Powles etal., 2004). During this period, Fgf10 expression is first observed in neural tissue in the ventral part of the posterior hindbrain (Fig. 1E; Alvarez et al., 2003).

As placode formation proceeds, Fgf8 disappears from the placodal ectoderm, but some transcripts are still observed in the ventral surface ectoderm, pharyngeal endoderm and intervening mesoderm between 8 to $12 \mathrm{ss}$ (Fig. 1F; Ladher et al., 2005; Park et al., 2006). Shortly before placode invagination at $10 \mathrm{ss}$, Fgf3 transcripts are now also seen in the pharyngeal endoderm (Mahmood et al., 1996; McKay et al., 1996). In the neural domain relevant to inner ear induction, Fgf3 is maintained in the developing hindbrain with strongest expression observed in $r 5$ and $r 6$ while it becomes downregulated in the otic placode as it starts to invaginate around 12ss-13ss (Fig. 1F; McKay et al., 1996; Wright and Mansour, 2003a). During this period Fgf10expression is initiated in the invaginating placode and the pharyngeal endoderm, where in the latter case it also accompanies the expression of $\mathrm{Fgf3}$ and Fgfo (Pirvola et al., 2000; Alvarez et al.,
Fig. 1. Expression of FGFs during otic placode induction in chick and mouse. Schematic sections of embryos taken at the level where inner ear induction takes place in chicken and mouse. The presence of the different FGF members is indicated by the colour code at the bottom of the figure. (A,D) Expression of Fgfs in the endoderm and/or mesoderm is observed during the first phase of induction. (B,E) A second phase of induction is defined by the onset of Fgf expression in neural tissue together with the initiation of otic placode formation in the surface ectoderm. (C,F) Finally, a third phase is characterized by the completion of placode formation and the initiation of placode invagination, a period when expression of some Fgfs expressed during placode induction is still maintained whilst other Fgfs initiate their expression in the placode itself or in the endoderm. For details, see text. Abbreviations: e, endoderm; $m$, mesoderm; $n$, neural tube; op, otic placode; pp, preplacodal ectoderm. 
2003; Wright and Mansour, 2003a). While Fgf10transcripts in the mesenchyme have diminished, Fgf10 expression in the ventral part of $r 5$ and $r 6$ that flank the invaginating placode is still evident (Alvarez et al., 2003; Wright and Mansour, 2003a).

Mouse FGF15 has been identified as the ortholog of chick FGF19. Unlike Fgf19, Fgf15is not expressed in the mesoderm but in the neuroectoderm from 0ss throughout all phases of otic induction and placode formation. Moreover, it is detected in the preplacodal ectoderm at 8ss and the pharyngeal endoderm from 13ss onwards (Wright et al., 2004).

\section{Zebrafish, Medaka and Xenopus FGF expression}

In contrast to the situation in chicken and mouse where several Fgfs are expressed in a dynamic manner in the different tissues involved during otic induction, in the zebrafish only two FGF members have been detected during this process. Fgf3and Fgf8 are coexpressed in several tissues implicated in otic induction. At $50 \%$ epiboly Fgf3 is expressed in the germring, at $75 \%$ epiboly in the prechordal plate, followed by expression in the anlage of $r 4$ and the paraxial cephalic mesoderm by $80 \%$ epiboly and early segmentation stages (Phillips et al., 2001; Maroon et al., 2002; Nechiporuk et al., 2007). Fgf8 shows a very similar expression pattern to $F g f 3$ in the germring and $\mathrm{r} 4$, but is not observed in the prechordal plate until the 6ss (Phillips et al., 2001; Maves et al., 2002; Walshe etal., 2002). Weak detection of Fgf8in the paraxial cephalic mesoderm has been reported at $80 \%$ epiboly followed by stronger expression during early segmentation (Reifers et al., 2000; Thisse et al., 2001; Nechiporuk et al., 2007; Nikaido et al., 2007). The spatiotemporal expression domains of Fgf3 and Fgf8 have been graphically outlined in detail by Phillips et al. (2001) and Whitfield et al. (2002). While no evidence has been obtained for the expression of Fgf10 and Fgf19during early otic induction, both these FGFs have been detected in the otic placode itself (Miyake et al., 2005; Thisse and Thisse, 2005b).

In Medaka, Fgf3 and Fgf8 are also coexpressed in a stripe in the posterior hindbrain from the end of gastrulation (stage 18) until the 6ss (stage 21, Hochmann et al., 2007). In Xenopus, Fgf3 expression is present from the late gastrula (Stage 12,5) and throughout neurulation in r3, r4 and r5 (Lombardo et al., 1998). Fgf8expression has not been described in the posterior hindbrain during neurulation, but is present as a horseshoe-shaped stripe corresponding to the placodal region of neuroectoderm adjacent to the developing neural tube (Christen etal., 2003; Fletcher etal., 2006).

\section{Functional analysis of FGFs during inner ear induction}

The experimental evidence for the involvement of FGF members during early inner ear development is discussed below. Key experiments and their results are summarized in Table 1.

TABLE 1

\section{KEY EXPERIMENTS ADDRESSING FGF FUNCTION DURING INNER EAR INDUCTION}

\begin{tabular}{|c|c|c|}
\hline Type of experiment & Outcome & References \\
\hline \multicolumn{3}{|l|}{ Chicken } \\
\hline Loss-of-function of FGF8 (si RNA at stage 4) & Reduced or absent placode and Pax2 expression & Ladher et al., 2005. \\
\hline Ectopic FGF8 (beads, stage 5 mesoderm) & Induction of FGF19 & Ladher et al., 2005. \\
\hline $\begin{array}{c}\text { Ectopic FGF19 (beads, stage } 5 \text { ectoderm, stage } 7 \text { non-otic tissue } \\
\text { including neural tissue) }\end{array}$ & Induction of FGF3 and otic markers & Ladher et al., 2000. \\
\hline Inhibition of FGF receptor signalling (SU5402) & Block of Pax2 expression (until 4ss) & Martin and Groves, 2006. \\
\hline Loss of FGF3 and FGF19 in posterior hindbrain (RA-deficient quail) & Formation of otic vesicle unaffected & Kil et al., 2005. \\
\hline Expression of FGF3 and FGF19 in neural tube (RA-deficient quail) & Expansion of otic placode and ectopic otic vesicles & Kil et al., 2005. \\
\hline Loss-of-function of FGF3 in hindbrain or pharyngeal endoderm (si RNA at stage $\mathrm{HH} 8$ ) & Block of otic vesicle formation & Zelarayan et al., 2007. \\
\hline $\begin{array}{l}\text { Ectopic FGF3 } \\
\text { (viral overexpression in surface ectoderm and electroporation in hindbrain at } \mathrm{HH} 8 \text { ) }\end{array}$ & Formation of ectopic otic vesicles & $\begin{array}{l}\text { Vendrell et al., 2000; } \\
\text { Zelarayan et al., } 2007 .\end{array}$ \\
\hline \multicolumn{3}{|l|}{ Mouse } \\
\hline $\begin{array}{l}\text { Ectopic FGF10 (FGF3) } \\
\text { (hindbrain, transgenic) }\end{array}$ & Formation of ectopic otic vesicles & Alvarez et al., 2003. \\
\hline FGF3 knockout & Reduced size of otic vesicle & Mansour et al., 1993; Alvarez et al., 2003. \\
\hline FGF10 knockout & Reduced size of otic vesicle & Ohuchi et al., 2000. \\
\hline FGF15 knockout & Fomation of otic vesicles & Wright et al., 2004. \\
\hline FGF3/FGF10 double knockout & Loss of otic vesicle or microvesicles & Alvarez et al., 2003; Wright et al., 2003. \\
\hline FGF3/FGF8 double knockout (FGF8 hypomorph or conditional allele) & Loss of otic vesicle or microvesicles & Ladher et al., 2005; Zelarayan et al., 2007. \\
\hline \multicolumn{3}{|l|}{ Fish and Xenopus } \\
\hline Loss-of-function of FGF3 or FGF8 (morpholino injection, mutants) & Reduced size of otic vesicle and otic marker expression & $\begin{array}{l}\text { Phillips et al., 2001; Léger et al., 2002; } \\
\text { Maroon et al., 2002; Liu et al., } 2003 .\end{array}$ \\
\hline Transplantation of wild-type cells in hindbrain of FGF8 mutant at shield stage & Rescue of Pax2 expression & Léger et al., 2002. \\
\hline Ectopic FGF3 and FGF8 (RA treatment, plasmid) & Formation of ectopic otic vesicles & $\begin{array}{l}\text { Lombardo et al., 1998; Phillips et al., 2001; } \\
\text { Bajoghli et al., 2004; Phillips et al., 2004; } \\
\text { Solomon et al., 2004; Hans et al., } 2007 .\end{array}$ \\
\hline Loss-of-function of FGF3 and FGF8 (morpholino injections, mutants) & Loss of otic vesicle and otic marker expression & $\begin{array}{l}\text { Phillips et al., 2001; Léger et al., } \\
\text { 2002; Maroon et al., 2002; Liu et al., } 2003 .\end{array}$ \\
\hline Inhibition of FGF receptor signalling (SU5402) & Block of Pax2 expression and otic vesicle formation & Léger et al., 2002; Maroon et al., 2002. \\
\hline
\end{tabular}




\section{Functional analysis of FGFs in chicken inner ear induc- tion}

The FGF family members that have been shown to act first during chicken inner ear induction are FGF8 and FGF19. The functional significance of their expression domains in the endoderm and mesoderm, respectively and their interactions have been addressed in two studies by Ladher et al. (Ladher et al., 2000; Ladher et al., 2005). FGF8 has been shown to be capable of inducing FGF19 in mesoderm isolated at HH5 (Ladher et al., 2005). Vice versa, electroporation of siRNA directed against Fgf8 in $\mathrm{HH} 4$ embryos results in the loss of Fgf19expression at $\mathrm{HH} 7$ as well as loss of Pax2expression and placodal tissue by HH12-14. Loss of Pax2 expression can be rescued by FGF19 in explant cultures derived from embryos electroporated with siRNA directed against Fgf8. Therefore, Fgf8expression in the endoderm is sufficient and necessary for Fgf19expression in the mesoderm and suggests that FGF8 acts via FGF19 during otic induction.

The involvement of FGF signalling between $0-4 \mathrm{ss}(\mathrm{HH} 6-8)$ has also been tested by blocking FGF receptors with SU5402 resulting in a loss of some (e.g. Pax2) but not all otic markers (Martin and Groves, 2006). Vice versa, FGF2, which is able to activate several isoforms of the four FGF receptors, induces otic markers in isolated non-otic ectoderm at this stage. In contrast, FGF19 by itself is not able to induce otic markers in presumptive otic or nonotic regions at these stages, but does so in the presence of neural tissue (Ladher et al., 2000; Martin and Groves, 2006). Fgf3 is present in mesodermal and neural tissue during otic induction and is induced by FGF19, but its sufficiency to induce otic markers at these stages has not yet been tested (Ladher et al., 2000; Kil et al., 2005).

Wnt8c is expressed in the neuroectoderm overlying Fgf19 expressing mesoderm at $\mathrm{HH} 7$ and is induced by FGF19 in unspecified stage 5 ectoderm. Wnt8c on the other hand induces Fgf3and weak expression of otic markers in isolated presumptive otic ectoderm at $\mathrm{HH} 7$, whereas the combination of both Wnt8c and FGF19 together induce strong expression of otic markers (Ladher et al., 2000). From these results it was proposed that FGF19 from the mesoderm and Wnt8c from neural tissue act as synergistic signals for otic induction. The influence of Fgf3, Fgf19 and $W n t 8 c$ expression on otic induction has also been recently addressed in vitamin A deficient (VAD) quails (Kil et al., 2005). In this experimental system, Fgf3 and Fgf19 expression is still present in the mesoderm whereas the posterior hindbrain is lost and expression of $F g f 3, F g f 19$ and $W n t 8 c$ has shifted caudally. Since the otic placode is still induced under these conditions, it was concluded that expression of these genes in the posterior hindbrain is not required during otic induction, whereas the expression of Fgf3and Fgf19in the mesoderm may be necessary. Nevertheless, the posterior hindbrain still has inducing activity stimulating otic placode formation since VAD embryos have a caudally expanded otic placode, possibly due to the posteriorly shifted expression domains of Fgf3, Fgf19 and Wnt8c in the neighbouring neural tube (Kil et al., 2005). Taken together, an alternative interpretation of the study by Ladher etal. (2000) might involve FGF19 and FGF3, with FGF3 being induced by both FGF19 and Wnt8c as the factors initially responsible for otic induction. However, the sufficiency or necessity for Fgf3expression in the mesoderm for otic induction has not yet been ad- dressed. Likewise, the necessity for Fgf19 during otic induction remains to be analysed by directly blocking its expression in the mesoderm using e.g. siRNA-mediated gene knockdown.

The role of FGF3 during early inner ear development has however been studied at slightly later stages, at $\mathrm{HH} 8$ (5ss). At this stage the placodal precursors that are initially specified as lens at HH6 (Bailey et al., 2006) have already acquired otic properties and the specification of the otic placode has occurred as assessed by Pax2expression (Groves and Bronner-Fraser, 2000). First, the necessity for Fgf3expression in the neural tube for early inner ear information has been addressed by siRNA-mediated knockdown of Fgf3 in vivo(Zelarayan etal., 2007). At HH8, strong expression of Fgf3is observed in the posterior hindbrain (Mahmood et al., 1995) and knockdown of its expression blocks the transition from the otic placode to the otic vesicle (Zelarayan et al., 2007). These results appear to confirm similar observations made when Fgf3expression was blocked in explants at HH10 (Represa et al., 1991). At this stage the otic placode has already completed its formation and is commited to form an otic vesicle (Groves and Bronner-Fraser, 2000). Invagination of the otic placode was blocked by antibodies which were raised against an epitope present in the chicken FGF3 protein (Represa et al., 1991). Similar results were obtained in the presence of anti-sense oligonucleotides directed against $F g f 3$, although the sequences used were based on human FGF3 and thus contained several mismatches (Mahmood et al., 1995). Nevertheless, there is now considerable evidence that expression of $\mathrm{Fgf3}$ in the hindbrain from $\mathrm{HH} 8$ onwards appears to be required for otic placode invagination. Likewise, results from the ectopic overexpression of Fgf3in the surface ectoderm and neural tube at $\mathrm{HH} 8$ in vivoin the intact embryo support this hypothesis (Vendrell et al., 2000; Zelarayan et al., 2007). These experiments lead to an increase in the size of the endogenous otic placode and in addition, to the formation of ectopic otic placodes and vesicles in a broad area of the surface ectoderm (Vendrell et al., 2000), reflecting a widespread competence to respond to FGF3. Although these experiments do not exclude an indirect action of FGF3 on otic placode formation via other signals, e.g. in the neighbouring mesoderm, they most likely mimic the action of FGF3 from its natural sources at this stage (e.g. the posterior hindbrain) by stimulating the formation of ectopic otic placodes in the competent surface ectoderm. Interestingly, isolated non-otic ectoderm at $\mathrm{HH} 8$ and earlier (0-4ss) induces otic but not neural or mesodermal markers upon treatment with FGF2, further suggesting a direct action of the FGF signal on the ectoderm (Martin and Groves, 2006). Finally, knockdown of Fgf3 in the pharyngeal endoderm at $\mathrm{HH} 8$ also blocks placode invagination (Zelarayan et al., 2007). Thus both hindbrain- and endoderm-derived FGF3 is required for this process.

The potency of the FGF3 signal is also underlined by overexpressing FGF2, FGF8 or FGF10 at HH8 in the surface ectoderm of the intact embryo, which fail to induce ectopic placodes (Vendrell et al., 2000; Y. Alvarez and T.S., unpublished observations). However, implantation of FGF2 beads into the mesoderm close to the future otic placode at $\mathrm{HH} 8$ in vivo results in the formation of small ectopic otic placodes at a low frequency (V. Vendrell and T.S., unpublished observations). Likewise, FGF2 induces small ectopic otic placodes close to the endogenous otic vesicle upon implantation of beads at $\mathrm{HH} 10$ to $\mathrm{HH} 11$ (Adamska et 
al., 2001). This activity may again reflect the capacity of FGF2 to activate isoforms of all four FGF receptors and thus also to promote ectopic otic placode formation although to a much lesser extent than FGF3. Both FGF2 and FGF8 beads implanted into the mesoderm also increase otic marker gene expression and the size of the normal otic vesicle (Adamska et al., 2001). These results may reflect a patterning function for FGF signalling in the otic vesicle, which has also been suggested in zebrafish (Leger and Brand, 2002).

\section{Functional analysis of FGFs in mouse inner ear induc- tion}

The sufficiency of FGFs to induce ectopic otic placodes from the developing hindbrain has been tested in transgenic mice (Alvarez et al., 2003). Different FGF family members were misexpressed in $\mathrm{r} 3$ and overexpressed in $\mathrm{r} 5$ from the 3ss stage onwards (Theil et al., 1998; Alvarez et al., 2003). Misexpression of Fgf3, whose endogenous onset of expression in the hindbrain coincides with this stage (Wright and Mansour, 2003a) shows only a very limited capacity to induce ectopic otic vesicles (Alvarez et al., 2003). In contrast, misexpression of Fgf10, whose endogenous onset of expression in the hindbrain takes place slightly later at the 5ss, leads consistently to the formation of ectopic vesicles next to $r 3-r 5$ that also express some otic markers (Alvarez et al., 2003). Interestingly, more recent analysis of these transgenic animals has revealed that ectopic Fgf8expression accompanies Fgf10 misexpressing embryos but not following ectopic expression of $F g f 3$, indicating a positive role for FGF8 during the induction of ectopic otic placodes (Zelarayan et al., 2007). However, misexpression of Fgf8 in r3 and r5 leads to early embryonic lethality and thus prevents the analysis of transgenic embryos during otic induction (Alvarez et al., 2003 and unpublished observations). Similarly, null mutants for Fgf8 have revealed an essential role for this gene during gastrulation and consequently show early embryonic lethality (Meyers et al., 1998). Thus, so far it has been difficult to assign a specific role for the early expression pattern of Fgf8 in the mesoderm and endoderm at E7-E8 during otic induction (Ladher et al., 2005). Mouse mutants carrying a hypomorphic or a conditional allele which is inactivated mosaically from E7 onwards next to Fgf8 null alleles form otic vesicles (Ladher et al., 2005; Zelarayan et al., 2007). Therefore, so far no evidence has been obtained for a unique requirement for FGF8 during inner ear induction. Tissue-specific inactivation of Fgfo in the mesoderm or endoderm may circumvent the early lethality and reveal the unique requirements of FGF8 in these tissues during inner ear induction.

Null mutants for Fgf3 or Fgf10 form otic vesicles albeit reduced in size (Ohuchi et al., 2000; Alvarez etal., 2003; Wright and Mansour, 2003b). Fgf3 mutants may also show a more ventrally localized otic vesicle and alterations in expression of otic markers in a variable manner
(Wright and Mansour, 2003a; Ladher et al., 2005). In humans, homozygous mutations in FGF3 that are likely to result in nonfunctional proteins are associated with a new form of syndromic deafness characterized by inner ear agenesis (Tekin et al., 2007). Due to the absence of inner ear structures in the patients it has been suggested that inner ear development is disturbed at a very early stage.

Mouse mutants for the FGF receptor $2 \mathrm{IIlb}$ isoform, to which FGF3 and FGF10 bind with high affinity also develop smaller otic vesicles (Pirvola et al., 2000). Finally, although mouse FGF15 is sufficient to induce otic markers in stage $4 / 5$ chicken rostral ectoderm, Fgf15 null mouse mutant embryos form normal otic vesicles (Wright et al., 2004). Likewise, inner ear phenotypes are absent in null mutants for FGF receptor 4 to which FGF15 binds with high affinity (Weinstein et al., 1998).

In contrast to mouse mutants that lack single members of the FGF gene family, FGF double mutants have been much more informative in demonstrating the roles of FGFs during inner ear induction thus revealing considerable redundancy between family members. Homozygous null mutant embryos for both Fgf3and Fgf10 either entirely lack otic vesicles or show the formation of microvesicles (Alvarez et al., 2003; Wright and Mansour, 2003a). Furthermore, the microvesicles formed are ventralised and more distantly located from the neural tube compared to control embryos (Figure 2A,C; Alvarez et al., 2003; Wright and Mansour, 2003a). Some of the microvesicles express otic genes whereas others show reduced or absent staining of otic markers, including Pax2and DIx5(Alvarez etal., 2003; Wright and Mansour, 2003a). Occasionally, development of these microvesicles continues to later stages of inner ear development, where defects in the dorsal
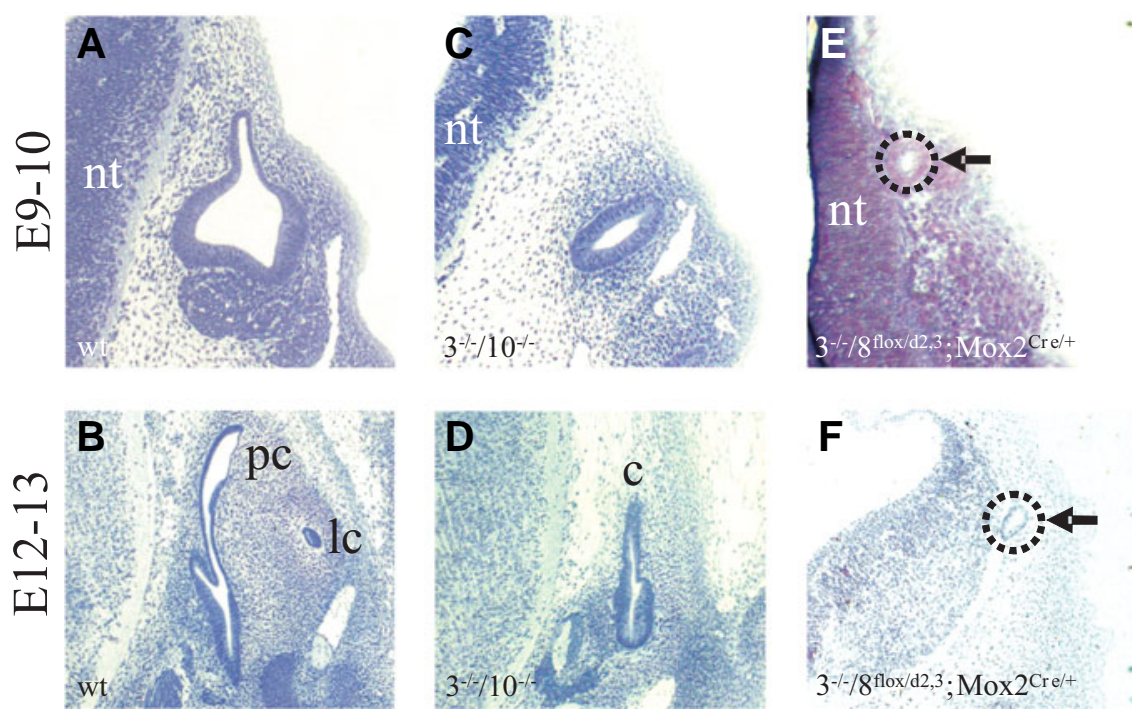

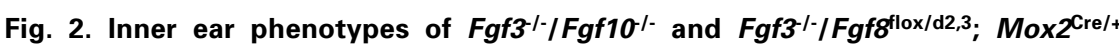
mutant mouse embryos. Sections through the developing inner ear of wild-type (A,B),

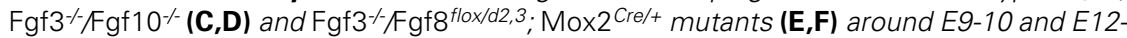
13. $(\mathbf{A}, \mathbf{C})$ Compared to wild-type embryos the otic vesicle is smaller and in a more ventral and distal position from the neural tube (nt) in $\mathrm{Fgf3}^{-/}$Fgf10 $\%$ mutants. (B,D) During otic vesicle differentiation Fgf3 $\%$ Fgf10\% embryos only form a single semicircular canal (c) whereas the posterior (pc), lateral (Ic) and anterior semicircular canals (not shown) are observed in wild-type embryos. (E,F) Fgf3 ${ }^{-1} / \mathrm{Fgf8}^{\mathrm{flox} / \mathrm{d} 2,3}$; $\mathrm{Mox}^{\mathrm{Cre} /+}$ mutants form microvesicles that are often found in a dorsal position in close proximity to the neural tube (nt). 
vestibular part of the inner ear now become evident (Fig. 2D; Zelarayan et al. 2007). Similar defects can also be observed in mutant embryos homozygous null for Fgf3 and carrying one mutant Fgf10 null allele (Zelarayan et al., 2007). Placode formation and the expression of otic placode markers has been analysed at E8 in $\mathrm{Fgf3}^{/-} / \mathrm{Fgfl}^{\prime-}$ double mutants (Alvarez et al., 2003; Wright and Mansour, 2003a). Placodal tissue and several otic placode markers are still detected in these mutants. However, the dorsal part of the placodal ectoderm fails to form and thus the expression of otic markers including Pax2or D/x5is absent in this area or in some cases missing entirely throughout the placode (Alvarez et al., 2003; Wright and Mansour, 2003a).

Double mutants for Fgf3and Fgf8develop a similar phenotype to $\mathrm{Fgf3}^{/-} / \mathrm{FgflO}^{\prime-}$ double mutants (Ladher et al., 2005; Zelarayan et al., 2007). Mutant embryos carrying a hypomorphic and a null allele for Fgf8 on a homozygous null Fgf3 mutant background $\left(F g f 3^{\prime-} / F g f g^{\text {neo/neo }}\right)$ do not form otic vesicles and lack expression of otic markers in the dorsal part of the placodal ectoderm (Ladher et al., 2005). Interestingly, these mutants also show a downregulation of Fgf10 expression in the mesoderm during inner ear induction, indicating that $F g f 3$ and $F g f 8$ may be redundantly required for normal levels of Fgf10 expression. Similar to $\mathrm{Fgfl}^{\prime-}$ / Fgfoneo/neo mutants, embryos carrying a null allele and a mosaically deleted conditional allele for Fgf8 on a homozygous null

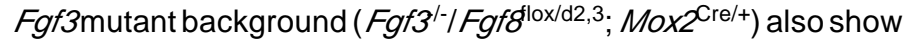
a severe phenotype that results in the formation of microvesicles which are in an abnormal dorsal position close to the neural tube (Fig. 2E; Zelarayan et al., 2007). These microvesicles show absence or abnormal expression of otic markers. At later stages these microvesicles fail to differentiate or a complete loss of otic tissue is observed (Fig. 2F; Zelarayan et al., 2007). Taken together, comparative phenotyping of $\mathrm{Fgf3}^{/-} / \mathrm{Fgfl}^{\prime-}$ and $\mathrm{Fgf3}$ Fgfodouble mutant combinations indicate that the latter mutants have a slightly more severe inner ear phenotype (Ladher et al., 2005; Zelarayan et al., 2007). This suggests a more pronounced role for FGF8 rather than FGF10 during inner ear induction, possibly due to the earlier and more widespread expression of Fgf8 in several tissues implicated during otic induction. Inactivation of both Fgf8and Fgf10 should result in an even more severe phenotype, because $F g f 8$ and $F g f 10$ are both expressed before Fgf3 and are likely to initiate otic induction as early endodermal and/or mesodermal signals.

Examination of the hindbrain of $\mathrm{Fgf3}^{/-} / \mathrm{Fgfl}^{/-}$and $\mathrm{Fgf3}^{/-}$ $F g f 8^{n e o / n e o ~ m u t a n t ~ e m b r y o s ~ s h o w e d ~ n o ~ c h a n g e s ~ i n ~ h i n d b r a i n ~}$ marker expression, indicating that FGFs do not act indirectly on otic induction by controlling hindbrain patterning as suggested in zebrafish (Wright and Mansour, 2003a; Ladher et al., 2005). Since high-affinity receptors for FGF3 and FGF10 are expressed in the preplacodal ectoderm, at least these two FGFs may act directly to establish expression of otic markers in the future otic placode (Wright and Mansour, 2003a). Interestingly, more recent studies have revealed that Fgf10 misexpression from the hindbrain is sufficient to rescue otic vesicle development in $\mathrm{Fgf3}^{/-1}$ Fgf10/- mutant embryos (Zelarayan et al., 2007). This shows that a FGF signal from neural tissue (the hindbrain) is able to reinstruct the placodal ectoderm in these mutants to form an otic vesicle. Since the formation of placodal tissue is only partially affected in $\mathrm{Fgf3}^{/-} / \mathrm{FgflO}^{\prime-}$ mutant embryos the initial steps of placode induction could be maintained by the expression of Fgfo which is present in various tissues during early otic induction (Ladher etal., 2005). Fgf3and Fgf10thus possibly reinforce and maintain inner ear induction initiated by Fgf8.

\section{Functional analysis of FGFs in zebrafish, medaka and Xenopus inner ear induction}

The effects of a loss of FGF3 or FGF8 on inner induction in zebrafish has been tested in mutants and by morpholino knockdown experiments. Using both approaches, a reduction in size of the otic vesicle combined with reduced or loss of expression of otic markers has been observed (Phillips et al., 2001; Leger and Brand, 2002; Maroon et al., 2002; Liu et al., 2003). The size of the otic vesicle in Fgf8 morphants appears slightly smaller than in Fgf3morphants, possibly due to a non-redundant requirement for FGF8 during hindbrain patterning influencing otic induction (Wiellette and Sive, 2004). The central role of the hindbrain during inner ear induction is also underlined by the fact that only wild-type hindbrain cells rescue Pax2 expression in Fgf8 mutant embryos in cell transplantation experiments (Leger and Brand, 2002). Furthermore, ectopic expression of Fgf3 or Fgf8 induces otic markers and formation of ectopic otic vesicles in zebrafish, Medaka and Xenopus (Lombardo etal., 1998; Phillips et al., 2001; Bajoghli et al., 2004; Hans et al., 2004; Phillips et al., 2004; Solomon et al., 2004). Initially, an indirect way for ectopic expression of both Fgf3 and Fgf8 was chosen by treating wild-type zebrafish with retinoic acid (Phillips et al., 2001). This leads to an expansion of the expression domains of Fgf3and Fgf8and of the otic marker Pax8 and results in the formation of ectopic otic vesicles. However, retinoic acid treatment may also have more pleiotropic effects in these experiments since the neural plate is posteriorized and the hindbrain is expanded (Phillips et al., 2001). More recently, experiments were used to demonstrate that FGFdependent otic induction by retinoic acid may also occur without perturbing patterning of the neural plate (Hans et al., 2007). Similarly, plasmid-mediated misexpression of Fgf3or Fgf8at the 8 cell stage leads to ectopic or expanded expression of the otic markers Pax8, Pax2a and D/x3b without expansion of the neural plate (Phillips et al., 2004; Solomon et al., 2004). To better control the timing of ectopic FGF expression during otic induction in embryos, heat-inducible promoters have been successfully used for Fgf8 in Medaka and more recently also in zebrafish (Bajoghli et al., 2004; Hans et al., 2007). In the latter case, early misexpression of Fgf8 until midgastrula stages was shown to actually reduce the amount of otic tissue, probably due to its effects on dorsoventral patterning that negatively affect expression of Foxi1 and $D / x 3 b$ that are required as competence factors for FGF-dependent otic induction (see below). In contrast, larger otic vesicles are obtained when Fgf8misexpression is carried out between the end of gastrulation and early segmentation stages, presumably due to the induction of a larger area of competent ectoderm to acquire an otic fate (Hans et al., 2007).

The redundant requirements for FGF3 and FGF8 for otic placode formation have been demonstrated in several studies (Phillips et al., 2001; Leger and Brand, 2002; Maroon et al., 2002; Liu et al., 2003). All studies agree that zebrafish mutants or morphants lacking both FGF3 and FGF8 show a severe loss or absence of otic tissue and markers. However, some discrepancies exist on the presence or absence of the earliest marker 
indicating otic fate, Pax8, in Fgf3/Fgf8double mutants. Whereas most studies report a severe reduction or absence of this marker (Phillips et al., 2001; Leger and Brand, 2002; Liu et al., 2003) one study reports the maintenance of normal Pax 8 expression in about half of the double mutant embryos (Maroon et al., 2002). In a related experiment, these authors also demonstrate unchanged expression of Pax8 upon blocking FGF receptor signalling with the inhibitor SU5402. In contrast, Leger and Brand (2002) note absence of Pax8 expression using even lower concentrations of SU5402 than those reported by Maroon et al. (2002). More consistently, both studies report absence of the otic marker Pax2 upon SU5402 treatment before segmentation, but differ again on the effects of SU5402 on Pax2maintenance at later stages (Leger and Brand, 2002; Maroon et al., 2002). The remaining otic tissue in some Fgf3/Fgf8 double mutants has been shown to consist of a few scattered placodal cells only, indicating that the capacity to form a placodal epithelium has been lost (Liu et al., 2003). In the cases of double mutant embryos where Pax 8 expression is not detected and morphological signs of otic placode formation are not observed one may conclude that a complete loss of otic placode induction has been achieved.

Loss of FGF3 and FGF8 also affects the development of the posterior hindbrain (Maves et al., 2002; Walshe et al., 2002) where targets of FGF receptor signalling are downregulated in the hindbrain and otic region (Maroon et al., 2002). Therefore, the hindbrain region where Fgf3 and Fgf8 are coexpressed plays an essential role for inner ear induction in zebrafish. However, both FGFs are also expressed in other tissues known to be involved during inner ear induction, such as the cephalic paraxial mesoderm (Mendonsa and Riley, 1999; Phillips et al., 2001; Thisse et al., 2001; Nechiporuk etal., 2007). Zebrafish mutants or morphants for one-eyed pinhead (oep) that lack mesoendodermal tissue underlying the otic placode show a loss of Fgf3 and Fgf8 in their mesoendodermal domains of expression (Phillips et al., 2001; Leger and Brand, 2002; Nechiporuk et al., 2007). In both cases hindbrain expression of $F g f 3$ and $F g f 8$ was not affected during inner ear induction. However, while one study reported normal expression of otic markers including Pax 8 (Leger and Brand, 2002), Phillips et al. (2001) showed that expression of this otic marker was reduced, indicating a possible requirement for FGF3 and/or FGF8 for inner ear induction outside of the hindbrain. Therefore, at present it is still unclear which expression domains of $F g f 3$ and $F g f 8$ are required for inner ear induction in zebrafish.

The model that envisages FGF signalling cooperating with Wnt8during otic placode induction, originally suggested in chicken has been analysed in more detail in zebrafish (Ladher etal., 2000; Phillips et al., 2004). Ectopic expression of Fgf3 or Fgf8 was shown to be sufficient to induce ectopic otic placodes in the absence of Wnt8. However, global ectopic expression of Wnt8 also induced ectopic otic tissue, but this effect was shown to depend on the expansion of Fgf3 and Fgf8 expression domains. Finally, otic induction and expression of Fgf3 and Fgf8 was delayed in Wnt8morphants. Since vice versa, Wnt8expression in the hindbrain is also reduced in Fgf3/Fgfo double mutants, the existence of a positive feedback loop has been postulated, that guarantees timely expression of $F g f 3$ and $F g f 8$ in the hindbrain which then act directly on preplacodal cells to induce the otic fate (Phillips et al., 2004).

Several studies have analysed the interaction of FGF3 and
FGF8 signalling with transcription factors expressed during inner ear induction in the preplacodal surface ectoderm. Foxi1 has been shown to be required for the induction of Pax8 expression mediated by FGF signalling (Hans et al., 2004; Solomon et al., 2004; Hans et al., 2007). Foxi1 has therefore been termed a competence factor for FGF3 and FGF8 that permits the acquirement of otic fate by preplacodal cells, as assessed by Pax 8 expression (Nissen et al., 2003; Hans et al., 2004; Solomon et al., 2004; Hans et al., 2007). On the other hand, Pax8 morphants have more profound defects during inner ear induction in a Fgf8 mutant background than in the presence of $F g f 3$ morpholinos, indicating once again a more dominant role for FGF8 compared to FGF3 (Wiellette and Sive, 2004; Mackereth et al., 2005). A second pair of competence factors for FGF signalling, $D / x 3 b$ and $D / x 4 b$, have been shown to be required for the proper initiation of Pax2a expression at a later stage (Hans et al., 2004; Mackereth et al., 2005). Sox9a expression has also been shown to depend on FGF signalling via the expression of Pax8 and later the maintenance of Sox9a expression depends on Pax2a (Hans et al., 2004). Finally, zebrafish Atoh1b, a homologue of Atoh 1 that is necessary for hair cell differentiation in the mouse (Woods et al., 2004) has recently been shown to be required in the preplacodal ectoderm in zebrafish (Millimaki et al., 2007). Atoh $1 b$ is coexpressed with Pax 8 in preplacodal ectoderm during early segmentation and requires FGF signalling. More detailed schemes of the interaction of transcription factors and FGF signalling during otic induction can be found in the studies of Hans et al., (2004), Solomon et al. (2004) and Millimaki et al. (2007).

In Xenopus, expression of a dominant negative FGF receptor has been shown to reduce Sox9expression (Saint-Germain et al., 2004). Morpholinos directed against Sox9lead to the absence of Pax8expression and otic vesicles are not formed. This lead to the suggestion that Sox9may be upstream of Pax8but may also be explained by a positive feedback loop between both genes to maintain each others expression (Liu et al., 2003; Hans et al., 2004).

\section{Summary and outlook}

The expression studies of different FGF members during inner ear induction confirm their sequential presence in endomesodermal and neural tissue during inner ear induction. Direct evidence for the necessity of a single FGF member during the initiation of inner ear induction (before otic specification) only currently exists for FGF8 in the chicken endoderm (Ladher et al., 2005). Moreover, the sufficiency and necessity of FGF8 to induce FGF19 in the overlying mesoderm indicates that this event represents an important step during chicken inner ear induction. However, at present direct evidence for the necessity of FGF19 for inner ear induction is lacking. Since Fgf3is induced by FGF19 and both are coexpressed in the mesoderm (Ladher et al., 2000; Kil et al., 2005) the necessity and sufficiency of FGF3 in the mesoderm for the early phase of inner ear induction is certainly worth testing.

The significance of the early mesodermal and/or endodermal expression of Fgf8 together with Fgf10 in mouse or Fgf3 in zebrafish during inner ear induction has not yet been directly addressed. Tissue-specific inactivation of Fgf8or Fgf10 in a Fgf3 homozygous null mutant background during this phase will provide useful information on the necessity of these expression 
domains for otic induction in the mouse. The second phase of inner ear induction (after otic specification and before otic commitment) is clearly defined by the conserved expression of Fgf3in the developing hindbrain in all vertebrates. Since in the mouse, Fgf3 expression is not present in endoderm or mesoderm at or before this stage, the effects on inner ear induction seen in $\mathrm{Fgfl}^{\prime / /} / \mathrm{Fgf10}$ I- and Fgf3/Fgfo double knockout mice have to be attributed entirely to the loss of hindbrain Fgf3expression. Interestingly, at this stage Fgf10 and Fgf19 which are initially expressed in the mesoderm are now present in the ventral part of the hindbrain in mouse and chicken, respectively, from where they may participate in otic induction (Ladher et al., 2000; Alvarez et al., 2003). Knockdown of Fgf3 in chick hindbrain interferes with placode invagination but more severe phenotypes may be obtained upon inactivation at an earlier stage (Zelarayan et al., 2007).

Fgf3/Fgf8 double mutants in zebrafish have a more severe phenotype than both $\mathrm{Fgf3}^{\prime-} / \mathrm{FgflO}^{\prime-}$ and $\mathrm{Fgf3} / \mathrm{Fgf8}$ double mutants in mouse. While the zebrafish mutants often completely lack placodal tissue (Phillips et al., 2001; Leger and Brand, 2002), mouse mutants usually still form some placodal ectoderm or microvesicles (Alvarez et al., 2003; Wright and Mansour, 2003a; Ladher et al., 2005). These phenotypes may be caused by a complete loss of Pax8 expression in the zebrafish double mutants, whereas this marker is reduced but still present in the double mouse mutants. In contrast, Pax2 expression which is activated after Pax8expression during otic development, is more consistently absent from placodal tissue in both zebrafish and mouse FGF double mutants. The residual Pax 8 expression at (and possibly before) E8 in mouse FGF double mutants may be sufficient for the formation of some placodal tissue. However, by analogy to the zebrafish, absence of Pax2 and $D / x$ genes then may also lead to the loss of responsiveness to FGF signalling in the murine otic placode and thus to the formation of microvesicles or complete absence of otic tissue at later stages.

It has been suggested that the ventral part of the placodal ectoderm, which continues to express some otic genes in FGF double mouse mutants, may contribute to the epibranchial placodes (Groves, 2005). In zebrafish it has recently been shown that Sox3 defines the common primordium of the otic and epibranchial placodes (Sun etal., 2006; Nikaido etal., 2007). This Sox3-positive primordium then segregates into a Pax2a-positive medial area and a Pax2a-negative lateral area, giving rise to the otic and epibranchial placodes, respectively. Disruption of FGF signalling in FGF zebrafish mutants or by using SU5402 leads to a loss of Sox3 expression and a failure to form the epibranchial placodes (Sun et al., 2006; Nechiporuk et al., 2007; Nikaido et al., 2007). It will thus be interesting to further define the differential requirements for the induction of the otic placode versus the epibranchial placode, for example by examining expression of Sox3and the formation of the epibranchial placode in mouse FGF double mutants.

\section{Acknowledgments}

I thank all present and former members of our laboratory, especially Maria Teresa Alonso, Yolanda Alvarez, Elena Dominguez-Frutos, Victor Vendrell and Laura Zelarayan for having provided data used for this review and Mark Maconochie for comments on the manuscript. Supported by the Spanish MEC (BFU2004-860) and the DFG (SFB 444).

\section{References}

ADAMSKA, M., HERBRAND, H., ADAMSKI, M., KRUGER, M., BRAUN, T. and BOBER, E. (2001). Fgfs control the patterning of the inner ear but are not able to induce the full ear program. Mech Dev 109: 303-13.

ALVAREZ, Y., ALONSO, M.T., VENDRELL, V., ZELARAYAN, L.C., CHAMERO, P., THEIL, T., BOSL, M.R., KATO, S., MACONOCHIE, M., RIETHMACHER, D. et al. (2003). Requirements for fgf3 and fgf10 during inner ear formation. Development 130: 6329-38.

BAILEY, A.P. and STREIT, A. (2006). Sensory organs: making and breaking the pre-placodal region. Curr Top Dev Bio/72: 167-204.

BAILEY, A.P., BHATTACHARYYA, S., BRONNER-FRASER, M. and STREIT, A. (2006). Lens specification is the ground state of all sensory placodes, from which fgf promotes olfactory identity. Dev Cel/11: 505-17.

BAJOGHLI, B., AGHAALLAEI, N., HEIMBUCHER, T. and CZERNY, T. (2004). An artificial promoter construct for heat-inducible misexpression during fish embryogenesis. Dev Bio/271: 416-30.

BAKER, C.V. and BRONNER-FRASER, M. (2001). Vertebrate cranial placodes i. Embryonic induction. Dev Bio/232: 1-61.

BOTTCHER, R.T. and NIEHRS, C. (2005). Fibroblast growth factor signaling during early vertebrate development. Endocr Rev 26: 63-77.

CHRISTEN, B., BECK, C.W., LOMBARDO, A. and SLACK, J.M. (2003). Regeneration-specific expression pattern of three posterior hox genes. Dev Dyn226: 34955.

FLETCHER, R.B., BAKER, J.C. and HARLAND, R.M. (2006). Fgf8 spliceforms mediate early mesoderm and posterior neural tissue formation in xenopus. Development 133: 1703-14.

GROVES, A.K. (2005). The induction of the otic placode. In Development of the inner ear, (ed. KELLEY, M.W., WU, D.K., POPPER, A.N., FAY., R.R.). Springer, New York, pp. 10-42.

GROVES, A.K. and BRONNER-FRASER, M. (2000). Competence, specification and commitment in otic placode induction. Development 127: 3489-99.

HAMBURGER, V. and HAMILTON, H.L. (1992). A series of normal stages in the development of the chick embryo. 1951. Dev Dyn 195: 231-72.

HANS, S., CHRISTISON, J., LIU, D. and WESTERFIELD, M. (2007). Fgf-dependent otic induction requires competence provided by foxi1 and dlx3b. BMC Dev Biol7: 5.

HANS, S., LIU, D. and WESTERFIELD, M. (2004). Pax8 and pax2a function synergistically in otic specification, downstream of the foxi1 and dlx3b transcription factors. Development 131: 5091-102.

HIDALGO-SANCHEZ, M., ALVARADO-MALLART, R. and ALVAREZ, I.S. (2000). Pax2, otx2, gbx2 and fgf8 expression in early otic vesicle development. Mech Dev 95: 225-9.

HOCHMANN, S., AGHAALLAEI, N., BAJOGHLI, B., SOROLDONI, D., CARL, M. and CZERNY, T. (2007). Expression of marker genes during early ear development in medaka. Gene Expr Patterns 7: 355-62.

KARABAGLI, H., KARABAGLI, P., LADHER, R.K. and SCHOENWOLF, G.C. (2002). Comparison of the expression patterns of several fibroblast growth factors during chick gastrulation and neurulation. Anat Embryol(Berl)205: 36570.

KIL, S.H., STREIT, A., BROWN, S.T., AGRAWAL, N., COLLAZO, A., ZILE, M.H. and GROVES, A.K. (2005). Distinct roles for hindbrain and paraxial mesoderm in the induction and patterning of the inner ear revealed by a study of vitamina-deficient quail. Dev Bio/285: 252-71.

LADHER, R.K., ANAKWE, K.U., GURNEY, A.L., SCHOENWOLF, G.C. and FRANCIS-WEST, P.H. (2000). Identification of synergistic signals initiating inner ear development. Science 290: 1965-7.

LADHER, R.K., WRIGHT, T.J., MOON, A.M., MANSOUR, S.L. and SCHOENWOLF, G.C. (2005). Fgf8 initiates inner ear induction in chick and mouse. Genes Dev 19: 603-13.

LEGER, S. and BRAND, M. (2002). Fgf8 and fgf3 are required for zebrafish ear placode induction, maintenance and inner ear patterning. Mech Dev 119: 91108. 
LIU, D., CHU, H., MAVES, L., YAN, Y.L., MORCOS, P.A., POSTLETHWAIT, J.H. and WESTERFIELD, M. (2003). Fgf3 and fgf8 dependent and independent transcription factors are required for otic placode specification. Development 130: 2213-24.

LOMBARDO, A., ISAACS, H.V. and SLACK, J.M. (1998). Expression and functions of fgf-3 in xenopus development. Int J Dev Bio/42: 1101-7.

MACKERETH, M.D., KWAK, S.J., FRITZ, A. and RILEY, B.B. (2005). Zebrafish pax8 is required for otic placode induction and plays a redundant role with pax2 genes in the maintenance of the otic placode. Development 132: 371-82.

MAHMOOD, R., KIEFER, P., GUTHRIE, S., DICKSON, C. and MASON, I. (1995). Multiple roles for fgf-3 during cranial neural development in the chicken. Development 121: 1399-410.

MAHMOOD, R., MASON, I.J. and MORRISS-KAY, G.M. (1996). Expression of fgf3 in relation to hindbrain segmentation, otic pit position and pharyngeal arch morphology in normal and retinoic acid-exposed mouse embryos. Anat Embryol (Berl) 194: 13-22.

MAROON, H., WALSHE, J., MAHMOOD, R., KIEFER, P., DICKSON, C. and MASON, I. (2002). Fgf3 and fgf8 are required together for formation of the otic placode and vesicle. Development 129: 2099-108.

MARTIN, K. and GROVES, A.K. (2006). Competence of cranial ectoderm to respond to fgf signaling suggests a two-step model of otic placode induction. Development 133: 877-87.

MAVES, L., JACKMAN, W. and KIMMEL, C.B. (2002). Fgf3 and fgf8 mediate a rhombomere 4 signaling activity in the zebrafish hindbrain. Development 129 : 3825-37.

MCKAY, I.J., LEWIS, J. and LUMSDEN, A. (1996). The role of fgf-3 in early inner ear development: An analysis in normal and kreisler mutant mice. Dev Bio/174: 370-8.

MENDONSA, E.S. and RILEY, B.B. (1999). Genetic analysis of tissue interactions required for otic placode induction in the zebrafish. Dev Bio/206: 100-12.

MEYERS, E.N., LEWANDOSKI, M. and MARTIN, G.R. (1998). An fgf8 mutant allelic series generated by cre- and flp-mediated recombination. Nat Genet 18: 136-41.

MILLIMAKI, B.B., SWEET, E.M., DHASON, M.S. and RILEY, B.B. (2007). Zebrafish atoh1 genes: Classic proneural activity in the inner ear and regulation by fgf and notch. Development 134: 295-305.

MIYAKE, A., NAKAYAMA, Y., KONISHI, M. and ITOH, N. (2005). Fgf19 regulated by hh signaling is required for zebrafish forebrain development. Dev Bio/288: 259-75.

NECHIPORUK, A., LINBO, T., POSS, K.D. and RAIBLE, D.W. (2007). Specification of epibranchial placodes in zebrafish. Development 134: 611-23.

NIKAIDO, M., DOI, K., SHIMIZU, T., HIBI, M., KIKUCHI, Y. and YAMASU, K. (2007). Initial specification of the epibranchial placode in zebrafish embryos depends on the fibroblast growth factor signal. Dev Dyn 236: 564-71.

NISSEN, R.M., YAN, J., AMSTERDAM, A., HOPKINS, N. and BURGESS, S.M. (2003). Zebrafish foxi one modulates cellular responses to fgf signaling required for the integrity of ear and jaw patterning. Development 130: 2543-54.

NORAMLY, S. and GRAINGER, R.M. (2002). Determination of the embryonic inner ear. J Neurobio/53: 100-28.

OHUCHI, H., HORI, Y., YAMASAKI, M., HARADA, H., SEKINE, K., KATO, S. and $\mathrm{ITOH}$, N. (2000). Fgf10 acts as a major ligand for fgf receptor 2 iiib in mouse multi-organ development. Biochem Biophys Res Commun 277: 643-9.

PHILLIPS, B.T., BOLDING, K. and RILEY, B.B. (2001). Zebrafish fgf3 and fgf8 encode redundant functions required for otic placode induction. Dev Bio/235: 351-65.

PHILLIPS, B.T., STORCH, E.M., LEKVEN, A.C. and RILEY, B.B. (2004). A direct role for fgf but not wnt in otic placode induction. Development 131: 923-31.

PIRVOLA, U., SPENCER-DENE, B., XING-QUN, L., KETTUNEN, P., THESLEFF, I., FRITZSCH, B., DICKSON, C. and YLIKOSKI, J. (2000). Fgf/fgfr-2(iiib) signaling is essential for inner ear morphogenesis. J Neurosci20: 6125-34.

POWLES, N., MARSHALL, H., ECONOMOU, A., CHIANG, C., MURAKAMI, A., DICKSON, C., KRUMLAUF, R. and MACONOCHIE, M. (2004). Regulatory analysis of the mouse fgf3 gene: Control of embryonic expression patterns and dependence upon sonic hedgehog (shh) signalling. Dev Dyn 230: 44-56.
REIFERS, F., WALSH, E.C., LEGER, S., STAINIER, D.Y. and BRAND, M. (2000). Induction and differentiation of the zebrafish heart requires fibroblast growth factor 8 (fgf8/acerebellar). Development 127: 225-35.

REPRESA, J., LEON, Y., MINER, C. and GIRALDEZ, F. (1991). The int-2 protooncogene is responsible for induction of the inner ear. Nature 353: 561-3.

REUSS, B. and VON BOHLEN UND HALBACH, O. (2003). Fibroblast growth factors and their receptors in the central nervous system. Cell Tissue Res 313: 139-57.

SAINT-GERMAIN, N., LEE, Y.H., ZHANG, Y., SARGENT, T.D. and SAINTJEANNET, J.P. (2004). Specification of the otic placode depends on sox9 function in xenopus. Development 131: 1755-63.

SOLOMON, K.S., KWAK, S.J. and FRITZ, A. (2004). Genetic interactions underlying otic placode induction and formation. Dev Dyn 230: 419-33.

STOLTE, D., HUANG, R. and CHRIST, B. (2002). Spatial and temporal pattern of fgf-8 expression during chicken development. Anat Embryol (Berl) 205: 1-6.

SUN, S.K., DEE, C.T., TRIPATHI, V.B., RENGIFO, A., HIRST, C.S. and SCOTTING, P.J. (2006). Epibranchial and otic placodes are induced by a common fgf signal, but their subsequent development is independent. Dev Biol. 303: 675686.

TEKIN, M., HISMI, B.O., FITOZ, S., OZDAG, H., CENGIZ, F.B., SIRMACI, A., ASLAN, I., INCEOGLU, B., YUKSEL-KONUK, E.B., YILMAZ, S.T. et al. (2007). Homozygous mutations in fibroblast growth factor 3 are associated with a new form of syndromic deafness characterized by inner ear agenesis, microtia and microdontia. Am J Hum Genet 80: 338-344.

THEIL, T., FRAIN, M., GILARDI-HEBENSTREIT, P., FLENNIKEN, A., CHARNAY, P. and WILKINSON, D.G. (1998). Segmental expression of the epha4 (sek-1) receptor tyrosine kinase in the hindbrain is under direct transcriptional control of krox-20. Development 125: 443-52.

THISSE, B., PFLUMIO, S. and FÜRTHAUER, M., LOPPIN, B., HEYER, V., DEGRAVE, A., WOEHL, R., LUX, A., STEFFAN, T., CHARBONNIER, X.Q. AND THISSE, C. (2001). Expression of the zebrafish genome during embryogenesis. ZFIN Direct Data Submission.

THISSE, B. and THISSE, C. (2005a). Functions and regulations of fibroblast growth factor signaling during embryonic development. Dev Bio/287: 390-402.

THISSE, C. and THISSE, B. (2005b). High throughput expression analysis of zfmodels consortium clones. ZFIN Direct Data Submission.

VENDRELL, V., CARNICERO, E., GIRALDEZ, F., ALONSO, M.T. and SCHIMMANG, T. (2000). Induction of inner ear fate by fgf3. Development 127: 2011-9.

WALSHE, J., MAROON, H., MCGONNELL, I.M., DICKSON, C. and MASON, I. (2002). Establishment of hindbrain segmental identity requires signaling by fgf3 and fgf8. Curr Biol12: 1117-23.

WEINSTEIN, M., XU, X., OHYAMA, K. and DENG, C.X. (1998). Fgfr-3 and fgfr-4 function cooperatively to direct alveogenesis in the murine lung. Development 125: 3615-23.

WHITFIELD, T.T., RILEY, B.B., CHIANG, M.Y. and PHILLIPS, B. (2002). Development of the zebrafish inner ear. Dev Dyn 223: 427-58.

WIELLETTE, E.L. and SIVE, H. (2004). Early requirement for fgf 8 function during hindbrain pattern formation in zebrafish. Dev Dyn 229: 393-9.

WOODS, C., MONTCOUQUIOL, M. and KELLEY, M.W. (2004). Math1 regulates development of the sensory epithelium in the mammalian cochlea. NatNeurosci 7: 1310-18.

WRIGHT, T.J., LADHER, R., MCWHIRTER, J., MURRE, C., SCHOENWOLF, G.C. and MANSOUR, S.L. (2004). Mouse fgf15 is the ortholog of human and chick fgf19, but is not uniquely required for otic induction. Dev Bio/269: 264-75.

WRIGHT, T.J. and MANSOUR, S.L. (2003a). Fgf3 and fgf 10 are required for mouse otic placode induction. Development 130: 3379-90.

WRIGHT, T.J. and MANSOUR, S.L. (2003b). Fgf signaling in ear development and innervation. Curr Top Dev Bio/57: 225-59.

ZELARAYAN, L., VENDRELL, V., ALVAREZ, Y., DOMINGUEZ-FRUTOS, E., THEIL, T., ALONSO, M.T., MACONOCHIE, M. AND SCHIMMANG, T. (2007). Differential requirements for FGF3, FGF8 and FGF10 during inner ear development. Dev Bio/DOI: 10.1016/j.ydbio.2007.05.033

Published Online: 27th August 2007 


\section{Related, previously published Int. J. Dev. Biol. articles}

See our Special Issue Ear Development edited by Fernando Giraldez and Bernd Fritzsch at: http://www.ijdb.ehu.es/web/contents.php?vol=51\&issue=6-7

Analysis of Netrin 1 receptors during inner ear development

Tanja Matilainen, Maarja Haugas, Jordan A. Kreidberg and Marjo Salminen

Int. J. Dev. Biol. (2007) 51: 409-414

Cell proliferation during the early compartmentalization of the Xenopus laevis inner ear

Quincy A. Quick and Elba E. Serrano

Int. J. Dev. Biol. (2007) 51: 201-210

Functional analysis of FGF3 during zebrafish inner ear development

V Vendrell, D Gimnopoulos, T Becker, T Schimmang Int. J. Dev. Biol. (2001) 45: S105-S106

Role of Raf kinases during inner ear development. C Sanz, Y Leon, M Garcia-Gil and I Varela-Nieto Int. J. Dev. Biol. (1996) 40: S83-S84

p75 and Trk oncoproteins expression is developmentally regulated in the inner ear of human embryos.

E Vazquez, I San José, J Naves, J A Vega and J Represa Int. J. Dev. Biol. (1996) 40: S77-S78

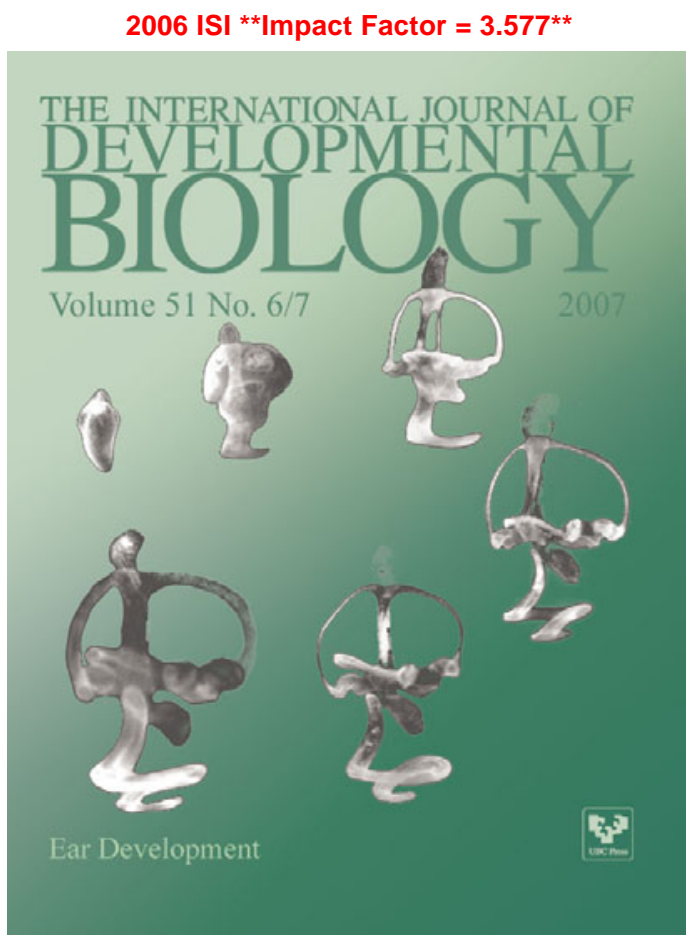

